\title{
Ressonância não linear de uma bússola em campos magnéticos
}

(Non-linear resonance of a compass in magnetic fields)

\author{
S.I. Finazzo, A.L. Tamborilo e A.A.P. Suaide ${ }^{1}$ \\ Instituto de Física, Universidade de São Paulo, São Paulo, SP, Brasil \\ Recebido em 21/8/2009; Revisado em 17/11/2009; Aceito em 11/12/2009; Publicado em 17/1/2011
}

\begin{abstract}
Fenômenos oscilatórios e ressonantes são explorados em vários cursos experimentais de física. Em geral os experimentos são interpretados no limite de pequenas oscilações e campos uniformes. Neste artigo descrevemos um experimento de baixo custo para o estudo da ressonância em campo magnético da agulha de uma bússola fora dos limites acima. Nesse caso, termos não lineares na equação diferencial são responsáveis por fenômenos interessantes de serem explorados em laboratórios didáticos.
\end{abstract}

Palavras-chave: ressonância em campos magnéticos, sistemas oscilatórios, equações diferenciais não-lineares.

Oscillatory and resonant phenomena are usually explored in many experimental physics courses. These experiments are generaly interpreted in the regime of small angle oscillation and uniform fields. In this paper we describe a low cost resonance experiment to explore physics phenomena out of this regime. In such case, non-linear terms in the differential equation are responsible for interesting physics behavior of the oscillatory system.

Keywords: resonance in magnetic field, oscillatory systems, non-linear differential equations.

\section{Introdução}

Fenômenos oscilatórios são explorados em muitos cursos experimentais de física. Experimentos típicos são os do estudo de pêndulos simples, sistemas massa-mola, circuitos RLC, dentre outros. Uma característica comum ma maioria destes experimentos é a sua interpretação no limite de pequenas oscilações e campos uniformes, como no caso do pêndulo simples, ou das características próximas ao ideal, como no caso do estudo de circuitos RLC. Nessas situações, as equações diferenciais que descrevem o comportamento do sistema físico são lineares nas suas coordenadas. Sistemas físicos descritos por equações diferenciais lineares correspondem apenas a um subconjunto dos fenômenos naturais. Com o avanço da computação e de técnicas de cálculo numérico o estudo de sistemas não lineares tem despertado um interesse cada vez maior, tanto na pesquisa básica como em aplicações diversas. Nesse caso, é interessante que estes fenômenos possam ser explorados e interpretados corretamente nos cursos de física, em especial, nos cursos experimentais.

Neste artigo descrevemos um experimento simples, de baixo custo, que pode ser realizado em laboratórios didáticos de física. O experimento consiste no estudo da ressonância de uma bússola comum na presença de campos magnéticos. Quando inserida em um campo

\footnotetext{
${ }^{1}$ E-mail: suaide@if.usp.br.

magnético, ocorre a interação entre esse campo e o momento de dipolo magnético da bússola, fazendo com que, na situação de equilíbrio, a bússola se alinhe a esse campo. No período de transição há oscilação da bússola. A frequência de oscilação, bem como o seu amortecimento, dependem das características da bússola e da intensidade de campo magnético.

O experimento proposto é dividido basicamente em duas etapas, cada uma delas correspondendo a duas semanas de aula. Na primeira etapa, temos como objetivo caracterizar uma bússola. Isso é feito a partir da medida dos seus parâmetros físicos, como momento de inércia, momento de dipolo magnético e coeficiente de atrito entre a agulha da bússola e seu suporte, similar ao experimento descrito na Ref. [1]. Na segunda, a bússola é posta a oscilar de forma forçada. Nesse caso, ocorre o fenômeno de ressonância, no qual, dependendo das condições experimentais, a oscilação da bússola possui amplitude máxima. Para evidenciar efeitos não lineares, realizamos tomadas de dados em grandes oscilações angulares (tipicamente até 50-60²) com campos magnéticos não uniformes. A equação diferencial que descreve o movimento da agulha da bússola nessa condição é construída e resolvida numericamente. As previsões decorrentes deste cálculo são comparadas aos dados obtidos e os fenômenos observados são interpretados de acordo. 
Esse trabalho está dividido da seguinte forma: inicialmente descreveremos os aspectos teóricos necessários para compreensão do fenômeno de oscilação e ressonância de uma bússola em campo magnético. Em seguida apresentamos o arranjo experimental utilizado, suas características e cuidados necessários. A seguir descrevemos, em detalhes, cada uma das etapas de aquisição e análise de dados que foram realizadas. Por fim, apresentamos nossas conclusões e comentários finais.

\section{Aspectos teóricos}

Uma bússola pode ser caracterizada pelo seu momento de dipolo magnético $(\boldsymbol{\mu})$ [2]. Na presença de um campo magnético (B) essa bússola está sujeita a um torque [2] dado por

$$
\tau=\mu \times \mathbf{B} .
$$

Supondo que os campos magnéticos sejam coplanares com a bússola, ou seja, os torques resultantes promovam apenas a rotação da agulha da bússola em torno do seu ponto de apoio, podemos reduzir o movimento da sua agulha a um problema unidimensional e escrever a equação diferencial que descreve esse movimento como

$$
I \frac{d^{2}}{d t^{2}} \theta=\tau-\gamma \frac{d}{d t} \theta
$$

Sendo $I$ o momento de inércia da agulha da bússola. Consideramos que $\theta$ é o ângulo relativo entre a agulha da bússola e o campo magnético, conforme esquematizado na Fig. 1. O termo $\gamma \frac{d}{d t} \theta$ corresponde a um torque dissipativo, devido principalmente ao atrito da agulha com o seu suporte. Se o campo magnético for constante no tempo e espaço e se seu ângulo com a agulha for pequeno o suficiente para podermos aproximar $\sin \theta \sim \theta$, é possível reescrever a Eq. (2) como

$$
I \frac{d^{2}}{d t^{2}} \theta+\gamma \frac{d}{d t} \theta+\mu B \theta=0
$$

A Eq. (3) corresponde à equação de um oscilador harmônico amortecido cuja solução analítica é possível [3], de forma que

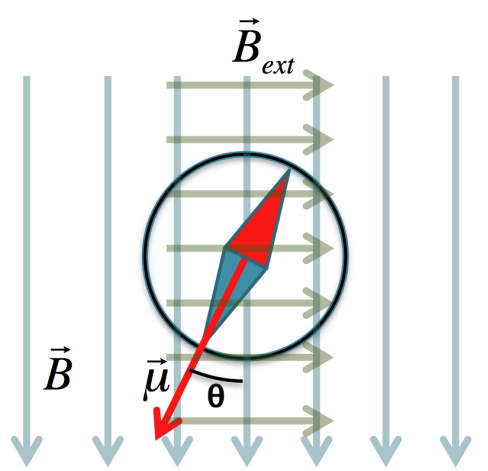

Figura 1 - Esquema de uma bússola na presença de campos magnéticos.

$$
\theta(t)=\theta_{0} e^{-\frac{\gamma}{2 I} t} \cos (\omega t),
$$

sendo $\theta_{0}$ o ângulo inicial entre a agulha da bússola e o campo magnético e $\omega$ a frequência de oscilação da bússola, que vale

$$
\omega=\sqrt{\frac{\mu}{I} B-\frac{\gamma^{2}}{4 I^{2}}} .
$$

Suponha agora que apliquemos um campo magnético oscilante harmonicamente no tempo, com frequência angular $\omega_{\text {ext }}$, de amplitude $B_{\text {ext }}$, perpendicular à B. Chamaremos este campo de campo perturbativo. Assim, no limite de pequenas oscilações, a Eq. (3) adquire um novo termo devido ao torque que este campo promove na agulha da bússola. Então

$$
I \frac{d^{2}}{d t^{2}} \theta+\gamma \frac{d}{d t} \theta+\mu B \theta+\mu B_{e x t} \cos \left(\omega_{e x t} t\right)=0 .
$$

A equação acima ainda pode ser resolvida analiticamente [3], e temos

$$
\theta(t)=\frac{\theta_{0}}{\sqrt{\left(\frac{\mu}{I} B-\omega_{e x t}^{2}\right)^{2}+\frac{\gamma^{2}}{I^{2}} \omega_{e x t}^{2}}} \sin \left(\omega_{e x t} t+\phi\right)
$$

com $\theta_{0}$ e $\phi$ constantes que satisfaçam as condições iniciais do movimento da agulha da bússola. Da Eq. (7), podemos notar que a agulha da bússola oscila com frequência igual à do campo perturbativo. Contudo, a amplitude de oscilação depende da frequência deste campo, sendo máxima quando a frequência do campo perturbativo satisfaça

$$
\omega_{e x t}=\sqrt{\frac{\mu}{I} B-\frac{\gamma^{2}}{2 I^{2}}}
$$

O fenômeno no qual a amplitude de oscilação atinge um valor máximo em uma frequência característica é denominado de ressonância [3]. Esta frequência (frequência de ressonância), no caso da agulha da bússola depende dos seus parâmetros geométricos (momento de dipolo magnético, momento de inércia e coeficiente de atrito), bem como do campo externo $\mathbf{B}$. Os resultados apresentados nas Eqs. (4), (5) e (8) podem ser utilizados para determinar estes parâmetros geométricos.

Porém, as condições necessárias para resolver analiticamente o problema da oscilação da agulha da bússola são idealizadas. Os ângulos de oscilação devem ser pequenos, para que as aproximações de $\sin \theta \sim \theta$ e $\cos \theta \sim 1$ sejam válidas, e que o campo magnético $\mathbf{B}$ seja uniforme. Essas condições nem sempre são possíveis experimentalmente. A aproximação de ângulos pequenos vale para até aproximadamente $15-20^{\circ}$, o que dificulta a medida experimental. A condição de campo uniforme é difícil de ser obtida com arranjos experimentais simples, como bobinas e imãs permanentes. Por isso, o problema 
experimental pode apresentar fenômenos interessantes de serem explorados devido à componentes não lineares na equação diferencial que descreve o movimento da agulha da bússola. Removendo essas simplificações, a Eq. (6) é reescrita como

$$
\begin{array}{r}
I \frac{d^{2}}{d t^{2}} \theta+\gamma \frac{d}{d t} \theta+\mu B(\theta) \sin (\theta)+ \\
\mu B_{\text {ext }}(\theta) \sin (\pi / 2-\theta) \cos \left(\omega_{\text {ext }} t\right)=0 .
\end{array}
$$

Note agora que, ambos os campos magnéticos possuem amplitudes que dependem do ângulo da bússola. A Eq. (9) não é mais uma equação diferencial linear e a sua solução analítica não é possível. Mas é possível resolvê-la numericamente e essa solução numérica será comparada com os dados experimentais adquiridos nesse experimento.

\section{Arranjo experimental}

Uma foto do arranjo experimental é mostrada na Fig. 2. Os equipamentos utilizados possuem baixo custo de fabricação e podem ser adquiridos facilmente no mercado nacional, tornando-o viável para laboratórios didáticos de física.

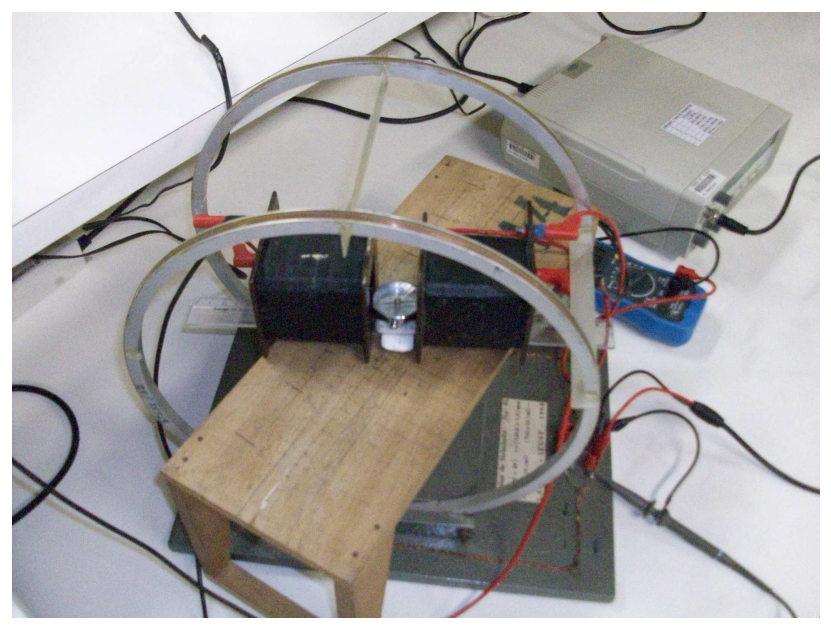

Figura 2 - Arranjo experimental utilizado para estudo da ressonância não linear de uma bússola em campo magnético.

O aparato consiste em duas bobinas de 1000 espiras cada uma (alguns alunos do curso utilizaram bobinas de 500 espiras, obtendo resultados qualitativamente semelhantes aos apresentados nesse trabalho). Essas bobinas são responsáveis pela geração do campo estático B. A distância entre as bobinas pode ser ajustada livremente, dependendo das características da bússola. Utilizamos distâncias de aproximadamente $3.5 \mathrm{~cm}$ entre essas bobinas, que suportam correntes de até aproximadamente $2 \mathrm{~A}$ e são conectadas a uma fonte de corrente contínua comum. Nessa configuração, o campo magnético entre as bobinas não é uniforme. Para parametrizar esse campo, utilizou-se o programa FEMM [4], que é um software livre, capaz de simular problemas eletromagnéticos com geometrias definidas pelo usuário. Na Fig. 3 é mostrado o campo magnético simulado em função do ângulo $\theta$ da agulha da bússola. Tomou-se como referência o campo na extremidade da agulha da bússola. A linha contínua representa um ajuste de uma função gaussiana, de largura $\sigma=(65.5 \pm 0.5)^{\circ}$, que será utilizada posteriormente na interpretação dos dados. Além disso, através dessa simulação, pode-se relacionar a amplitude de campo $(B)$ em $\theta=0$ com a corrente que flui nas bobinas $(i)$, através da relação de proporcionalidade $B=\beta i$. No nosso caso, $\beta=(85 \pm 6) \mathrm{G} / \mathrm{A}$.

O campo perturbativo é gerado por uma bobina de Helmholtz [5]. A utilização desta bobina torna o campo perturbativo uniforme, facilitando a interpretação dos resultados. Além disso, essa bobina gera um campo de magnitude pequena, se comparada à do campo estático. $\mathrm{Na}$ ausência de bobinas de Helmholtz, podemos utilizar bobinas comuns. Contudo, deve-se estar atento às intensidades dos campos perturbativos que, se muito intensos fazem com que a agulha da bússola gire indefinidamente, tornando impossível a tomada de dados. A bobina de Helmholtz é conectada a um gerador de frequências capaz de gerar ondas harmônicas com frequências entre 1 e $100 \mathrm{~Hz}$, de amplitude máxima de $10 \mathrm{~V}$, pico a pico.

A bússola utilizada consiste de uma bússola comum, encontrada facilmente no mercado. Notou-se, entretanto, que bússolas com agulhas mais leves e delgadas são mais apropriadas para o estudo proposto. Isso se deve ao fato de que essas agulhas, além do menor momento de inércia, possuem menor atrito no seu ponto de apoio gerando ressonâncias de amplitudes mais elevadas.

Na Tabela 1 apresentamos um quadro com as características físicas dos equipamentos utilizados. Além desses equipamentos foram utilizados réguas e paquímetros, para medidas geométricas do arranjo, multímetros, para medidas de tensões e correntes nas bobinas, osciloscópio, para monitoração da frequência do campo perturbativo, e uma máquina fotográfica digital com capacidade de filmadora, para registrar o movimento da agulha da bússola, quando necessário.

\section{Caracterização da bússola}

A primeira parte do experimento, que consiste tipicamente de duas semanas, é utilizada para caracterizar a bússola estudada. A caracterização da bússola é feita a partir da medida do seu momento de inércia, $I$, do seu momento de dipolo magnético, $\mu$, e da constante de amortecimento, $\gamma$. A medida do momento de inércia da bússola necessita da sua destruição, devido à necessidade de medir a massa e características geométricas da agulha. Por outro lado, de acordo com as expressões apresentadas na seção 2, é suficiente determinar as razões $\mu / I$ e $\gamma / I$. Isso pode ser realizado sem destruir a bússola. 
Tabela 1 - Características físicas do arranjo experimental.

\begin{tabular}{|c|c|c|c|c|}
\hline & $\begin{array}{l}\text { Raio da seção trans- } \\
\operatorname{versal}(\mathrm{cm})\end{array}$ & $\begin{array}{l}\text { Comprimento } \\
(\mathrm{cm})\end{array}$ & $\begin{array}{l}\text { Número de espiras em } \\
\text { cada bobina }\end{array}$ & $\begin{array}{l}\text { Resistência } \\
(\Omega)\end{array}$ \\
\hline Bobinas comuns & $1.6 \pm 0.2$ (interno) & & & \\
\hline & $3.2 \pm 0.2$ (externo) & $7.5 \pm 0.2$ & 1000 & $7.7 \pm 0.4$ \\
\hline Bobinas de Helmholtz & $14.9 \pm 0.1$ & $14.9 \pm 0.1$ & 28 & $0.742 \pm 0.015$ \\
\hline
\end{tabular}

De acordo com a expressão (8), podemos relacionar, na ressonância, a frequência do campo perturbativo com a magnitude do campo estático, tornando possível caracterizar a bússola. Porém, a expressão (8) é valida apenas nos limites de campo uniforme e pequenas oscilações. De acordo com a Fig. 3, no limite de pequenas oscilações $\left(\theta<10^{\circ}\right)$, o campo estático pode ser aproximado por um valor constante, satisfazendo assim, as condições necessárias.

O procedimento adotado foi o seguinte: Para cada valor de campo estático, ajustado a partir da corrente que flui sobre as bobinas, variar a frequência do campo perturbativo até que a amplitude de oscilação da agulha da bússola seja máxima, determinando a frequência de ressonância para esse dado valor de campo estático. Deve-se tomar o cuidado de ajustar a magnitude do campo perturbativo de tal forma que a amplitude de oscilação da bússola não ultrapasse $15-20^{\circ}$, conforme discutido anteriormente.

A Fig. 4 mostra a dependência da frequência de ressonância ao quadrado com a magnitude do campo magnético estático. Percebe-se claramente uma dependência linear entre essas variáveis. A partir de um ajuste linear, de acordo com a expressão (8), obtém-se que $\mu / I=(84 \pm 3) \mathrm{s}^{-2} \mathrm{G}^{-1}$ e $\gamma / I=(27 \pm 18) \mathrm{s}^{-1}$.

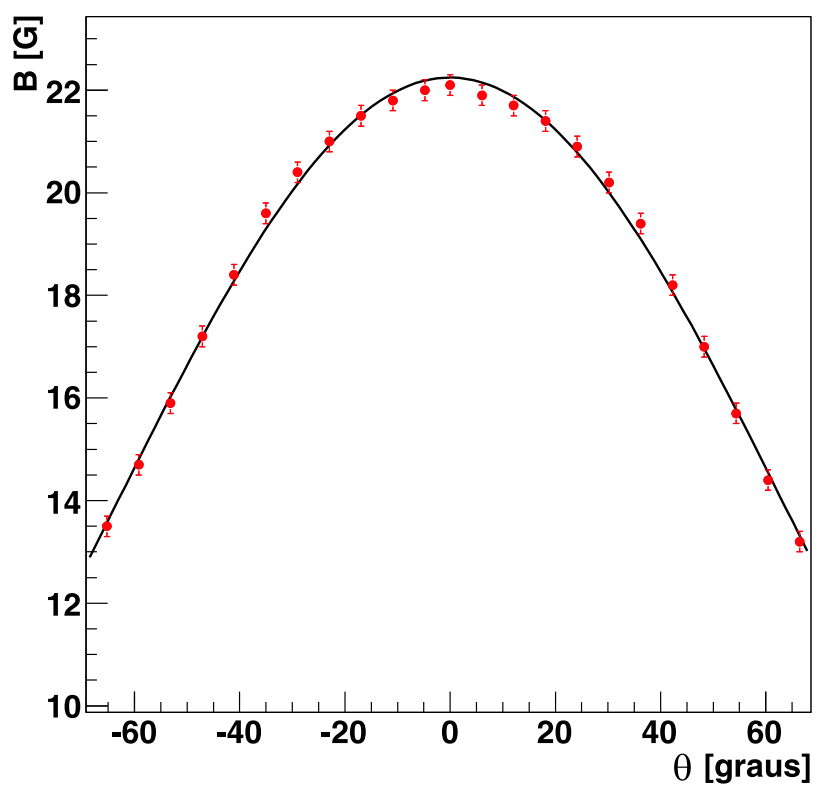

Figura 3 - Simulação do campo magnético das bobinas de 1000 espiras. A linha contínua corresponde a um ajuste gaussiano.

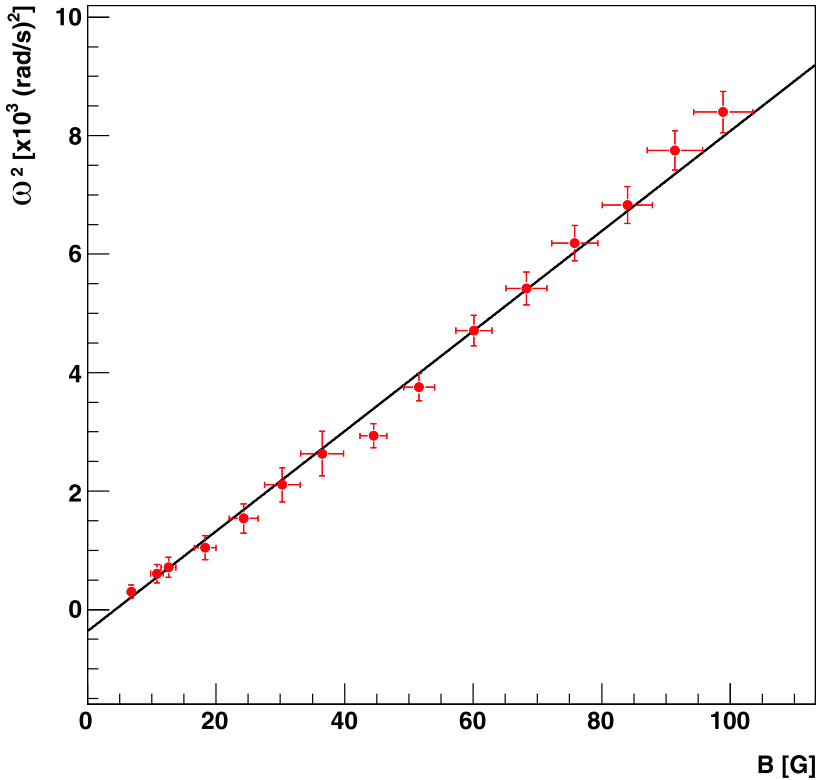

Figura 4 - Frequência de ressonância da agulha da bússola quadrática em função da magnitude do campo magnético estático. A linha contínua corresponde a um ajuste linear, de acordo com a expressão (8).

Note que, apesar desse método fornecer uma precisão razoável para a razão $\mu / I$, com incerteza da ordem de $4 \%$, a precisão na medida de $\gamma / I$ é pequena. Nesse caso, a incerteza é próxima de $60 \%$ da medida. Por outro lado, essa razão é importante nos estudos que serão realizados nas semanas seguintes do experimento, sendo necessária uma medida mais precisa.

Para realizar uma medida mais precisa de $\gamma / I$ colocamos a bússola a oscilar livremente, sem a presença de um campo perturbativo e com as bobinas desligadas. Assim, o campo estático é o próprio campo magnético terrestre local, considerado uniforme em toda a bússola. Nessas condições, o movimento da agulha da bússola corresponde a um movimento oscilatório amortecido, conforme a expressão (4), e foi filmado com uma câmera digital comum a uma taxa de 30 quadros por segundo. A posição angular da agulha da bússola foi determinada, quadro a quadro, utilizando o programa VideoPoint, versão $2.5[6]$.

A Fig. 5 mostra a posição da agulha da bússola em função do tempo. A linha contínua corresponde a um ajuste utilizando a expressão (4), de onde pode-se extrair que $\gamma / I=(1.02 \pm 0.04) \mathrm{s}^{-1}$, muito mais preciso que no procedimento anterior. A frequência de oscilação então vale $\omega=(0.51 \pm 0.02) \mathrm{rad} / \mathrm{s}$. Porém, por não conhecermos a amplitude do campo magnético 
local, não podemos determinar com precisão o valor de $\mu / I$.

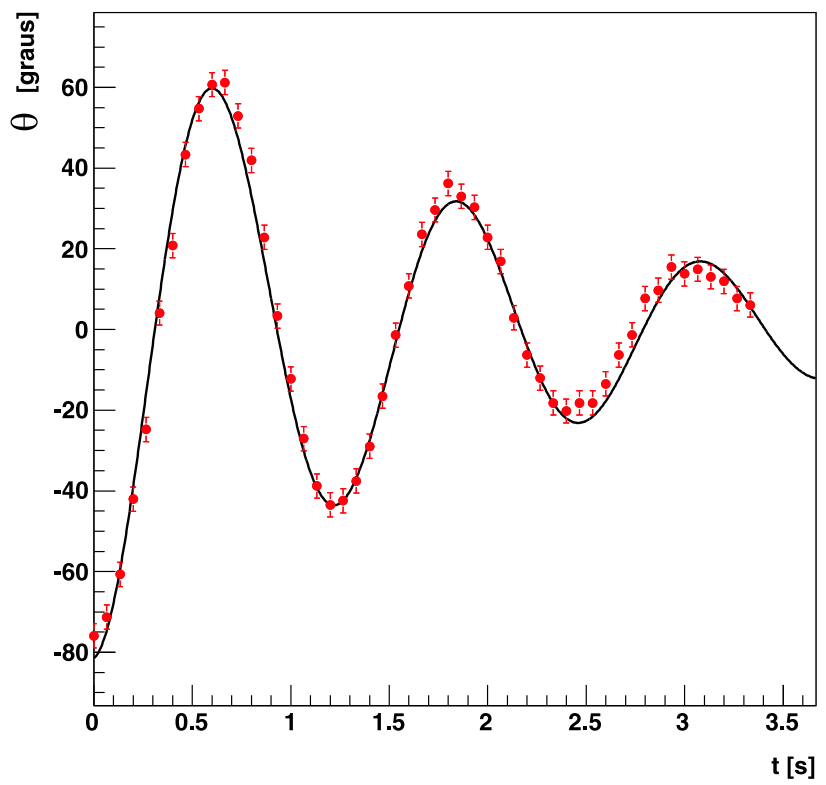

Figura 5 - Oscilação amortecida da bússola no campo magnético local. A linha contínua corresponde a um ajuste dos dados à expressão (4).

Do ponto de vista didático, o procedimento acima chama a atenção por tornar evidente que a precisão de uma medida pode depender fortemente do procedimento experimental adotado para realizá-la. O primeiro procedimento, apesar de adequado para medir $\mu / I$, é inadequado para medida de $\gamma / I$. O inverso ocorre no segundo procedimento. A comparação entre esses procedimentos e resultados obtidos é de grande ajuda nessa compreensão.

\section{Estudo da ressonância da bússola em campo magnético não uniforme}

A parte final do experimento consiste em investigar as características da ressonância da agulha da bússola para um campo estático bem determinado e não uniforme. Inicialmente, escolhe-se um valor de campo para as bobinas e, em seguida, mede-se a amplitude de oscilação em função da frequência do campo perturbativo em torno da frequência de ressonância. Essas medidas são realizadas de duas formas. A primeira delas no sentido de baixas para altas frequências e a segunda, no sentindo inverso. A motivação para esses dois procedimentos é de estudar a dependência da amplitude de oscilação com as condições iniciais do movimento já que, para uma dada frequência a ser medida, a condição inicial é o estado da bússola na frequência anterior. No caso de pequenas oscilações, não deve haver diferença entre um conjunto de medidas e outro, devido à linearidade da equação diferencial que rege o movimento da bússola. Já para grandes oscilações, onde o campo magnético estático não é uniforme, pode haver dependência com as condições iniciais do movimento.

A Fig. 6 mostra a dependência da amplitude de oscilação da agulha da bússola em função da frequência do campo perturbativo, mantendo o campo magnético estático fixo. Os círculos correspondem às medidas realizadas no sentido de baixas para altas frequências. Os quadrados correspondem ao sentido inverso. Notase, como esperado, que não há dependência da amplitude de oscilação com as condições iniciais do movimento da agulha da bússola. A linha contínua corresponde à previsão teórica para amplitude de oscilação (ver Eq. (7)) com os valores para $\mu / I$ e $\gamma / I$ obtidos na seção 4. A linha tracejada corresponde a um ajuste da expressão (7) aos dados. Nesse caso, foi obtido que $\mu / I=(82.8 \pm 0.2) \mathrm{s}^{-2} \mathrm{G}^{-1}$ e $\gamma / I=(1.3 \pm 0.3) \mathrm{s}^{-1}$. Notam-se a compatibilidade das curvas com os dados experimentais e a compatibilidade entre os valores ajustados àqueles obtidos anteriormente.

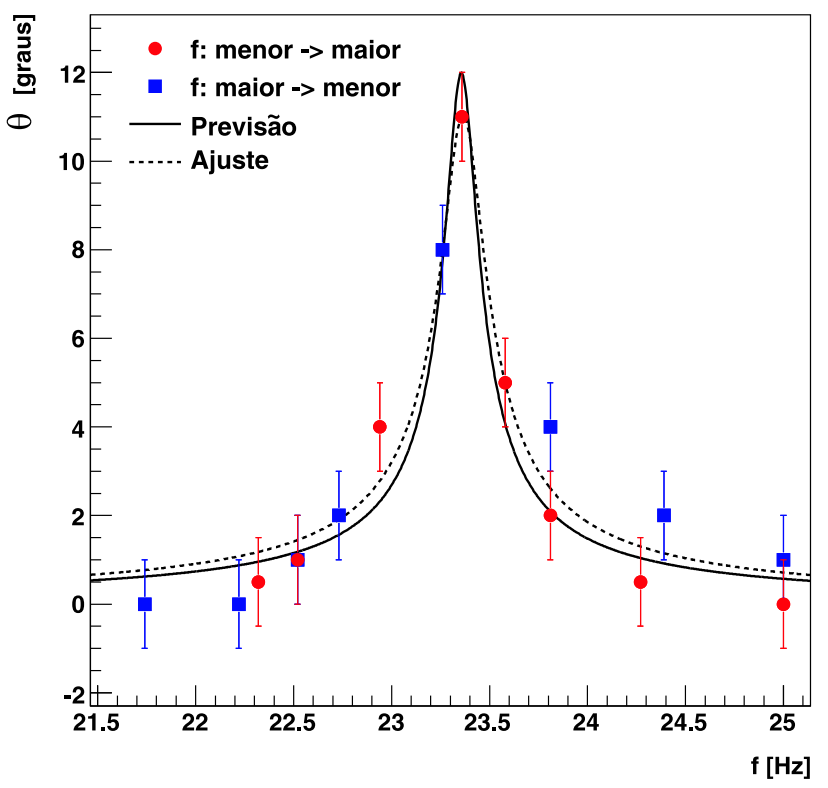

Figura 6 - Dependência da amplitude de oscilação da agulha da bússola em função da frequência do campo perturbativo. Ver texto para maiores detalhes.

Por outro lado, aumentando a corrente na bobina de Helmholtz, ou seja, aumentando a intensidade do campo perturbativo, em frequências próximas à frequência de ressonância da agulha da bússola, é comum que a mesma oscile com amplitude consideravelmente maior que $15-20^{\circ}$. Nesse caso, não podemos considerar o limite de pequenas oscilações, nem mesmo que o campo magnético estático é uniforme. A Fig. 7 mostra medidas de amplitude de oscilação em função da frequência do campo perturbativo nessa situação. O procedimento adotado para medida é o mesmo utilizado na tomada dos dados apresentados na Fig. 6. 


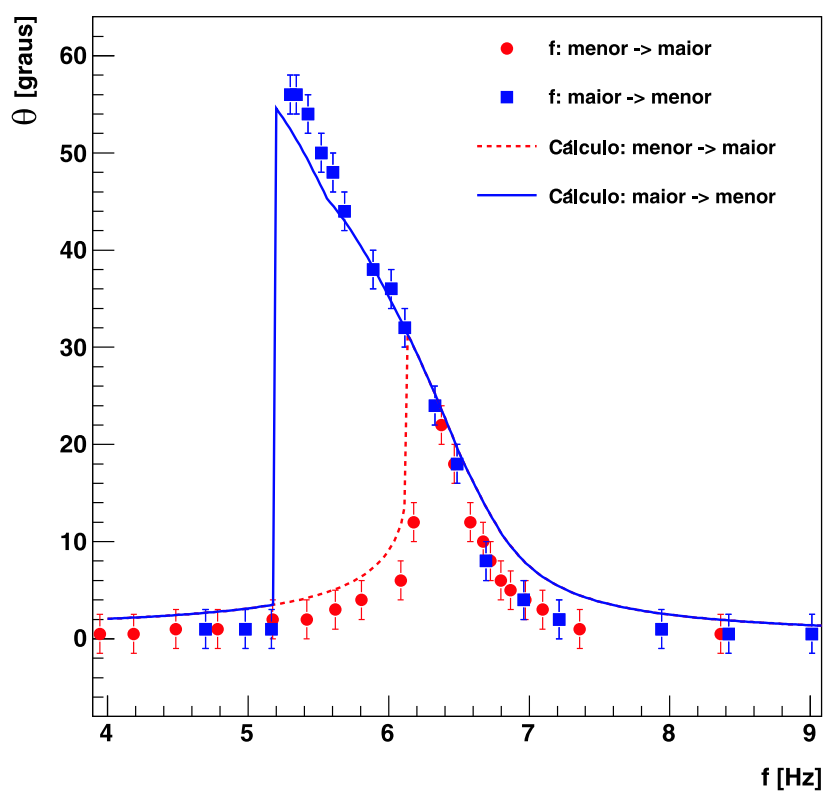

Figura 7 - Amplitude de oscilação em função da frequência para campos perturbativos elevados. Ver texto para mais detalhes.

O que podemos notar, primeiramente, é que a amplitude de oscilação não é função unívoca da frequência do campo perturbativo. No sentido de medidas de menores para maiores frequências é nítido que a curva é mais estreita e de menor amplitude máxima do que aquela no sentido inverso de medição. Como a diferença entre os conjuntos de dados se dá na condição inicial para cada frequência medida, é evidente a dependência da amplitude com as condições iniciais no movimento da agulha da bússola. Além disso, há um corte abrupto, em ambos sentidos de medida, na região de baixas frequências, ao contrário dos dados mostrados na Fig. 6, onde a amplitude aumenta (e diminui) suavemente.

Esses comportamentos podem ser interpretados, qualitativamente, como segue: Na medida em que a amplitude de oscilação aumenta, a bussola está sujeita a um campo estático médio menos intenso (ver Fig. 3). Como a frequência de ressonância depende do valor desse campo, a diminuição do mesmo diminui essa frequência. Os dados são, portanto, reflexo da mudança da frequência do campo perturbativo ao mesmo tempo em que a agulha da bússola muda sua frequência de ressonância. Em um conjunto de medidas, ao mesmo tempo que aumentamos a frequência perturbativa, diminuímos a frequência de ressonância. Assim, a agulha da bússola permanece em ressonância em uma faixa de frequências estreita. No outro caso, na medida em que diminuímos a frequência perturbativa, também diminuímos a frequência de ressonância. E a agulha da bússola permanece em ressonância por um intervalo de frequências maior.

As linhas contínuas na Fig. 7 representam a inte- gração numérica $^{2}$ da equação diferencial fora dos limites de pequenas oscilações, de acordo com a expressão (9). Nesse caso, $B(\theta)$ é dado por uma função Gaussiana. A Eq. (9) foi integrada utilizando o método de Runge-Kutta [7-8]. Os parâmetros utilizados foram aqueles obtidos na seção 4. Esses parâmetros foram variados em torno de uma incerteza, de modo a minimizar o $\chi_{\text {red }}^{2}$ entre os dados e o cálculo numérico. Os únicos parâmetros totalmente livres foram as amplitudes dos campos magnéticos. Para simular a situação experimental, a amplitude de cada frequência é calculada utilizando como condição inicial a amplitude para a frequência anteriormente calculada. Nota-se um bom acordo entre os dados e a solução numérica. O comportamento diferenciado entre as duas formas de medida é bem reproduzido.

Esse é um ponto particularmente interessante de ser explorado. Durante o experimento é pedido aos alunos apenas realizar a medida e compará-la com as previsões teóricas disponíveis. Em geral, não há a preocupação em manter as oscilações em pequenas amplitudes. Os alunos ficam surpresos ao observar que os dois conjuntos de medidas não são compatíveis entre si e não são compatíveis com a previsão teórica dada pela expressão (7). A partir disso discutimos as possíveis limitações no modelo ideal e discutimos os efeitos não lineares presentes no problema investigado.

\section{Conclusões}

Nesse trabalho apresentamos um experimento didático simples e de baixo custo para estudar efeitos de ressonância em uma bússola simples.

O experimento consiste de duas partes. A primeira parte corresponde à caracterização da bússola utilizada. É interessante investigar a necessidade de várias abordagens experimentais distintas para a total caracterização da mesma. Esse é um ponto importante no desenvolvimento e amadurecimento pelos alunos do método científico moderno.

A segunda parte consiste em estudar a ressonância da agulha da bússola em um campo estático bem determinado. Isso é feito em duas situações experimentais: no limite de pequenas oscilações os dados obtidos podem ser comparados à solução algébrica para um movimento harmônico forçado ideal e serve para confirmar os parâmetros característicos da bússola. Em seguida, as medidas são repetidas, desta vez em grandes oscilações. Nesse caso, efeitos não lineares, devidos principalmente à não uniformidade do campo magnético estático, tornam-se evidentes e passíveis de interpretação. Equações diferenciais não lineares são pouco exploradas em cursos experimentais de física, principalmente devido à complexidade interpretativa dos resultados obtidos. Nesse caso, além de inter-

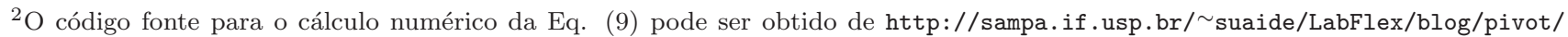
entry.php?id=99 ou através de contato de email com os autores desse trabalho. 
pretação simples, a solução numérica do problema é factível com recursos computacionais modestos.

\section{Agradecimentos}

Gostaríamos de agradecer ao corpo técnico dos laboratórios didáticos do Instituto de Física da Universidade de São Paulo pela disposição em ceder os equipamentos necessários à montagem desse experimento e pelo esforço empregado continuamente para manter os laboratórios didáticos em ótimo estado de funcionamento.

\section{Referências}

[1] G.T. Hageseth, Am. Journ. of Physics 37, 529 (1969.)

[2] H.M. Nussenzveig, Física Básica - Eletromagnetismo
(Edgard Blucher Ltda, São Paulo, 1997), v. 3, cap. 7, primeira edição.

[3] H.M. Nussenzveig, Física Básica - Fluídos, Oscilações e Ondas, Calor (Edgard Blucher Ltda, São Paulo, 1981), v. 2, cap. 4, segunda edição.

[4] D. Meeker, Programa FEMM (Finite Element Method Magnetics), v. 4.2 (2007).

[5] Wikipedia contributors, Helmholtz coil, disponível em http://en.wikipedia.org/w/index.php?title= Helmholtz $\{-\} \operatorname{coil}\{\backslash \&\} o l d i d=278411782$ (2009).

[6] Programa VideoPoint, v. 2.5, disponível em http: //www.1sw.com/videopoint/

[7] W.H. Press, S.A. Teukolsky, W.T. Vetterling and B.P. Flannery, Numerical Recipes in $\mathrm{C}++$ : The Art of Scientific Computing (Cambridge University Press, Cambridge, 2002), chap $16,2^{a}$ ed.

[8] M. Ducamp and A. Reverchon, Analyse en Turbo Pascal v. 5.5 et 6.0 (Eyrolles, Paris, 1991). 